\title{
Electric Potential of a Uniform Charged Disc in Whole by Method of Superposition
}

\author{
Tingting Liu \& Haibin Sun \\ Institute of Physics and Electronic Engineering, Taishan University \\ Tai' an 271021, China \\ E-mail: 1ttphd@163.com
}

The research is supported by the Education Reform Project of Taishan University (200905).

\begin{abstract}
It's a typical physical problem that the calculation of a uniformly charged circle and a uniformly charged disc. By solving partial differential equation under given boundary conditions, we obtained the electric potential of a uniformly charged circle. Using the method of superposition, the electric potential of a uniformly charged disc can be calculated.
\end{abstract}

Keywords: Uniformly charged circle, Charged disc, Electric potential, Method of superposition

\section{Introcuction}

It's a typical physical problem that the calculation of a uniformly charged circle and a uniformly charged disc. Zhou and Chen (2004) obtained the general expression of the spatial distribution of electrical field generated by a uniformly charged ring by using the equation of electric field strength, ploted the spatial distribution diagram for field magnitude and discussed the field at the central axis, in the distant area and on the plane of the ring. Li and Shan (2002) pointed out that $t$ legendre elliptic integral is needed to calculate the electric potentical on evenly electrified thin disc. They introduced the calculating method and pointed out that to choose proper polar coordinates can simplify calculating electric potenticals produced from electrified bodies. Cao and Dong (2005) progressed the elliptic integral with the method of numerical integration, and calculated the electric field intensity and the zeta potential on the plane embedded a uniformly charged circle.

In this paper, we discussed the spatial distribution of electrical field generated by a uniformly charged disc by using the method of superposition, and figured the three-dimensional potential distribution diagram of the charged disc.

\section{Spatial distribution of electric potential of a uniformly charged circle}

\subsection{General solution of the mathematical physics problem}

We consider a uniformly charged circle which radius is $R$ and the total charges is $q$ and calculate its spatial electric potential $u$. We establish the spherical coordinates frame, its origin of coordinates is the center of the ring and polar axis is the axis of the ring. See Fig. 1. There are no other charges in the space. The electric potential $u(r, \theta, \varphi)$ of point $P(r, \theta, \varphi)$ meets the Equation of Laplace. So we have

$$
\Delta u=0
$$

The solution of Eq. (1) can be written as a superposition in the normal way (Ken Riley and Michael Hobson, 2002, p12 14):

$$
u(r, \theta, \varphi)=\sum_{l=0}^{\infty} \sum_{m=-l}^{l}\left(A_{l}^{m} r^{l}+B_{l}^{m} \frac{1}{r^{l+1}}\right) P_{l}^{m}(\cos \theta)\left(C_{l}^{m} \cos m \varphi+D_{l}^{m} \sin m \varphi\right)
$$

The boundary condition that the electric potentials have axial symmetry implies that only $m=0$ terms are present. So, we have

$$
u(r, \theta)=\sum_{l=0}^{\infty}\left(A_{l} r^{l}+B_{l} \frac{1}{r^{l+1}}\right) P_{l}(\cos \theta)
$$


The sphere which radius is equal to the ring divides the space into two parts, one is inside the sphere (for $r<R$ ) and the other is outside the sphere ( for $r>R$ ).

Inside the sphere we require the solution to be finite at the origin and so $B_{l}=0$ for all $l$ in Eq. (3). Therefore, the electric potential inside the sphere is

$$
u_{i}(r, \theta)=\sum_{l=0}^{\infty} A_{l} r^{l} P_{l}(\cos \theta)
$$

Outside the sphere we require the solution to be bounded as $r$ tends to infinity and so in (3) we must have $A_{l}=0$ for all $l$. In this case, the electric potential outside the sphere is

$$
u_{e}(r, \theta)=\sum_{l=0}^{\infty} B_{l} \frac{1}{r^{l+1}} P_{l}(\cos \theta) .
$$

If only considering the natural boundary conditions, the coefficients of Eq. (4) and Eq. (5) can't be determined. It is necessary to use some certain electric potential to obtain the coefficients in the general solution.

\subsection{Speciall solution of the mathematical physics problem}

Considering the charge distribution of the thin ring and using Coulomb's law, we calculated the potential, $d u$, on the axis (for $\theta=0$ ) of the ring of a point charge, $d q$. See Fig. 2. The potential is

$$
d u=\frac{1}{4 \pi \varepsilon_{0}} \frac{d q}{\sqrt{R^{2}+r^{2}}},
$$

where $d q=\frac{q}{2 \pi R} d l$. We integrate Eq. (6) both sides and obtain

$$
u(r, 0)=\frac{q}{4 \pi \varepsilon_{0}} \frac{1}{\sqrt{R^{2}+r^{2}}} .
$$

This is the potential on the axis of the ring.

Taking into account the formula (Ken Riley and Michael Hobson, 2002, p143)

$$
(1+z)^{\alpha}=\sum_{n=0}^{\infty} \frac{\alpha(\alpha-1) \cdots(\alpha-n+1)}{n !} z^{n} .
$$

From Eq. (8), Eq. (7) can be written as a series form (for $r<R$ )

$$
\begin{gathered}
u_{i}(r, 0)=\frac{q}{4 \pi \varepsilon_{0}} \frac{1}{\mathrm{R}} \frac{1}{\sqrt{1+\left(\frac{r}{R}\right)^{2}}} \\
=\frac{q}{4 \pi \varepsilon_{0}} \frac{1}{\mathrm{R}}\left[1-\frac{1}{2}\left(\frac{r}{R}\right)^{2}+\frac{3}{8}\left(\frac{r}{R}\right)^{4}+\cdots+(-1)^{n} \frac{\left.\frac{1}{2}\left(\frac{1}{2}+1\right) \cdots\left(\frac{1}{2}+n-1\right)\left(\frac{r}{R}\right)^{2 n}+\cdots\right]}{n !}\right] \\
=\frac{q}{4 \pi \varepsilon_{0}} \frac{1}{\mathrm{R}}\left[1-\frac{1}{2}\left(\frac{r}{R}\right)^{2}+\frac{3}{8}\left(\frac{r}{R}\right)^{4}+\cdots+(-1)^{n} \frac{(2 n) !}{2^{n} n ! 2^{n} n !}\left(\frac{r}{R}\right)^{2 n}+\cdots\right],
\end{gathered}
$$


which can be simplied by using $P_{2 n}(0)=(-1)^{n} \frac{(2 n) !}{2^{n} n ! 2^{n} n !}$ (N. S. Koshlyakov, M. M. Smirnov and E. B. Gliner, 1964 , p205). So Eq. (9) can be written as

$$
\begin{aligned}
u_{i}(r, 0)=\frac{q}{4 \pi \varepsilon_{0}} \frac{1}{\mathrm{R}}\left[P_{0}(0)+P_{2}(0)\left(\frac{r}{R}\right)^{2}+P_{4}(0)\left(\frac{r}{R}\right)^{4}+\cdots+P_{2 n}(0)\left(\frac{r}{R}\right)^{2 n}+\cdots\right] & \\
& \text { for } 0<r<R .
\end{aligned}
$$

This is the potential distribution on the axis inside the sphere.

Similarly, we deduce the potential distribution on the axis outside the sphere,

$$
u_{e}(r, 0)=\frac{q}{4 \pi \varepsilon_{0}} \frac{1}{r} \frac{1}{\sqrt{1+\left(\frac{R}{r}\right)^{2}}}=\frac{q}{4 \pi \varepsilon_{0}} \frac{1}{r}\left[P_{0}(0)+P_{2}(0)\left(\frac{R}{r}\right)^{2}+P_{4}(0)\left(\frac{R}{r}\right)^{4}+\cdots+P_{2 n}(0)\left(\frac{R}{r}\right)^{2 n}+\cdots\right]
$$

for $r>R$.

Substituting Eq. (10) into Eq. (4), we have

$$
\begin{gathered}
u_{i}(r, 0)=\sum_{l=0}^{\infty} A_{l} r^{l} P_{l}(1) \\
=\frac{q}{4 \pi \varepsilon_{0}} \frac{1}{\mathrm{R}}\left[P_{0}(0)+P_{2}(0)\left(\frac{r}{R}\right)^{2}+P_{4}(0)\left(\frac{r}{R}\right)^{4}+\cdots+P_{2 n}(0)\left(\frac{r}{R}\right)^{2 n}+\cdots\right] \\
\text { for } 0<r<R .
\end{gathered}
$$

Appling the equation $P_{l}(1)=1$ (R. Courant, D. Hilbert, 1966, p503), and comparing the coefficients both side of the Eq. (11), we get

$$
\begin{gathered}
A_{1}=0, \quad A_{3}=0, A_{5}=0, \cdots, A_{2 \mathrm{n}+1}=0 ; \\
A_{0}=\frac{q}{4 \pi \varepsilon_{0}} \frac{1}{\mathrm{R}} P_{0}(0), A_{2}=\frac{q}{4 \pi \varepsilon_{0}} \frac{1}{\mathrm{R}} P_{2}(0) \frac{1}{R^{2}}, \cdots, A_{2 \mathrm{n}}=\frac{q}{4 \pi \varepsilon_{0}} \frac{1}{\mathrm{R}} P_{2 n}(0) \frac{1}{R^{2 n}} .
\end{gathered}
$$

Therefore, the distribution of the potential inside the sphere is

$$
\begin{array}{r}
u_{i}(r, \theta)=\frac{q}{4 \pi \varepsilon_{0}} \frac{1}{\mathrm{R}}\left[P_{0}(\cos \theta)+P_{2}(0)\left(\frac{r}{R}\right)^{2} P_{2}(\cos \theta)+P_{4}(0)\left(\frac{r}{R}\right)^{4} P_{4}(\cos \theta)+\cdots+P_{2 n}(0)\left(\frac{r}{R}\right)^{2 n} P_{2 n}(\cos \theta)+\cdots\right] \\
\text { for } \quad 0<r<R .
\end{array}
$$

Substituting Eq. (11) into Eq. (5), and comparing the coefficients, we obtain the distribution of the potential outside the sphere is

$$
\begin{array}{r}
u_{e}(r, \theta)=\frac{q}{4 \pi \varepsilon_{0}} \frac{1}{r}\left[P_{0}(\cos \theta)+P_{2}(0)\left(\frac{R}{r}\right)^{2} P_{2}(\cos \theta)+P_{4}(0)\left(\frac{R}{r}\right)^{4} P_{4}(\cos \theta)+\cdot \cdot+P_{2 n}(0)\left(\frac{R}{r}\right)^{2 n} P_{2 n}(\cos \theta)+\cdots\right] \\
\text { for } r>R .
\end{array}
$$

Eqs. (13) and (14) is the spatial potential distribution of the charged ring. Obviously, when $r=R$,

$$
u_{i}(R, \theta)=u_{e}(R, \theta)
$$




\section{Spatial distribution of electric potential of a uniformly charged disc}

We establish the spherical coordinates frame. The disc shown in Fig. 3 has a radius $a$ and carries a uniform surface charge $Q$. So that the charge per unit area $\sigma=\frac{Q}{\pi a^{2}}$. The following steps lead to the potential on the axis of the disc.

We consider the disk to be made out of an infinite number of infinitesimally thin rings. A single ring of radius $\rho$ and thickness $d \rho$ carries a total charge $d q=\frac{2 Q}{a^{2}} \rho d \rho$. The contribution of that ring to the disc's electric potential at point $p(r, \theta, \varphi)$ can be easily figured using Eqs. (13) and (14), replacing $R$ with $\rho$ and $q$ with $d Q:$

$$
\begin{gathered}
d u_{1}=\frac{Q}{2 \pi a^{2} \varepsilon_{0}} d \rho\left[P_{0}(\cos \theta)+P_{2}(0)\left(\frac{r}{\rho}\right)^{2} P_{2}(\cos \theta)+P_{4}(0)\left(\frac{r}{\rho}\right)^{4} P_{4}(\cos \theta)+\cdots+P_{2 n}(0)\left(\frac{r}{\rho}\right)^{2 n} P_{2 n}(\cos \theta)+\cdots\right] \\
\text { for } \quad \mathrm{r}<\rho, \\
d u_{2}=\frac{Q}{2 \pi a^{2} \varepsilon_{0}} \frac{\rho}{r} d \rho\left[P_{0}(\cos \theta)+P_{2}(0)\left(\frac{\rho}{r}\right)^{2} P_{2}(\cos \theta)+P_{4}(0)\left(\frac{\rho}{r}\right)^{4} P_{4}(\cos \theta)+\cdots+P_{2 n}(0)\left(\frac{\rho}{r}\right)^{2 n} P_{2 n}(\cos \theta)+\cdots\right] \\
\text { for } \quad \mathrm{r}>\rho .
\end{gathered}
$$

According to the superposition principle, we obtain the total potential produced by the charged disc by adding over all differential potentials.

Inside the sphere, the potential at point $p$ produced by the charged disc is

$$
\begin{gathered}
u_{i}(r, \theta)=\int_{0}^{r} d u_{2}+\int_{r}^{a} d u_{1} \\
=\int_{0}^{r} \frac{Q}{2 \pi a^{2} \varepsilon_{0}} \frac{\rho}{r}\left[P_{0}(\cos \theta)+P_{2}(0)\left(\frac{\rho}{r}\right)^{2} P_{2}(\cos \theta)+P_{4}(0)\left(\frac{\rho}{r}\right)^{4} P_{4}(\cos \theta)+\cdots+P_{2 n}(0)\left(\frac{\rho}{r}\right)^{2 n} P_{2 n}(\cos \theta)+\cdots\right] d \rho \\
+\int_{r}^{a} \frac{Q}{2 \pi a^{2} \varepsilon_{0}}\left[P_{0}(\cos \theta)+P_{2}(0)\left(\frac{r}{\rho}\right)^{2} P_{2}(\cos \theta)+P_{4}(0)\left(\frac{r}{\rho}\right)^{4} P_{4}(\cos \theta)+\cdots+P_{2 n}(0)\left(\frac{r}{\rho}\right)^{2 n} P_{2 n}(\cos \theta)+\cdots\right] d \rho \\
=\frac{Q}{2 \pi a \varepsilon_{0}} \frac{r}{a} \sum_{n=0}^{\infty}\left(\frac{1}{2 n+2}+\frac{1}{2 n-1}\right) P_{2 n}(0) P_{2 n}(\cos \theta)-\frac{Q}{2 \pi a \varepsilon_{0}} \sum_{n=0}^{\infty} \frac{1}{2 n-1}\left(\frac{r}{a}\right)^{2 n} P_{2 n}(0) P_{2 n}(\cos \theta)
\end{gathered}
$$$$
\text { for } r<a
$$

Outside the sphere, the potential at point $p$ produced by the charged disc is

$$
\begin{gathered}
u_{e}(r, \theta)=\int_{0}^{a} d u_{2} \\
=\int_{0}^{a} \frac{Q}{2 \pi a^{2} \varepsilon_{0}} \frac{\rho}{r}\left[P_{0}(\cos \theta)+P_{2}(0)\left(\frac{\rho}{r}\right)^{2} P_{2}(\cos \theta)+P_{4}(0)\left(\frac{\rho}{r}\right)^{4} P_{4}(\cos \theta)+\cdots+P_{2 n}(0)\left(\frac{\rho}{r}\right)^{2 n} P_{2 n}(\cos \theta)+\cdots\right] d \rho \\
=\frac{Q}{2 \pi a \varepsilon_{0}}\left[\frac{1}{2} \frac{a}{r} P_{0}(\cos \theta)+\frac{1}{4} P_{2}(0)\left(\frac{a}{r}\right)^{3} P_{2}(\cos \theta)+\frac{1}{6} P_{4}(0)\left(\frac{a}{r}\right)^{5} P_{4}(\cos \theta)+\cdots+\frac{1}{2 n+2} P_{2 n}(0)\left(\frac{a}{r}\right)^{2 n+1} P_{2 n}(\cos \theta)+\cdots\right] \\
=\frac{Q}{2 \pi a \varepsilon_{0}} \sum_{n=0}^{\infty} \frac{1}{2 n+2}\left(\frac{a}{r}\right)^{2 n+1} P_{2 n}(0) P_{2 n}(\cos \theta)
\end{gathered}
$$$$
\text { for } r>a \text {. }
$$

So, the distribution of the electric potential of the uniformly charged disc is 


$$
u(r, \theta)=\left\{\begin{array}{l}
\frac{Q}{2 \pi a \varepsilon_{0}} \frac{r}{a} \sum_{n=0}^{\infty}\left(\frac{1}{2 n+2}+\frac{1}{2 n-1}\right) P_{2 n}(0) P_{2 n}(\cos \theta)-\frac{Q}{2 \pi a \varepsilon_{0}} \sum_{n=0}^{\infty} \frac{1}{2 n-1}\left(\frac{r}{a}\right)^{2 n} P_{2 n}(0) P_{2 n}(\cos \theta), r<a, \\
\frac{Q}{2 \pi a \varepsilon_{0}} \sum_{n=0}^{\infty} \frac{1}{2 n+2}\left(\frac{a}{r}\right)^{2 n+1} P_{2 n}(0) P_{2 n}(\cos \theta), r>a .
\end{array}\right.
$$

When $r=a, u_{i}(a, \theta)=u_{e}(a, \theta)$. Using MATLAB, we can obtain the three-dimensional potential distribution image of the charged disc. See Fig. 4.

From Eq. (20), we can obtain the potential on the disc surface (for $\theta=\frac{\pi}{2}$ )

$$
u_{i}\left(r, \frac{\pi}{2}\right)=\frac{Q}{2 \pi a \varepsilon_{0}} \frac{r}{a} \sum_{n=0}^{\infty}\left(\frac{1}{2 n+2}+\frac{1}{2 n-1}\right) P_{2 n}(0)-\frac{Q}{2 \pi a \varepsilon_{0}} \sum_{n=0}^{\infty} \frac{1}{2 n-1}\left(\frac{r}{a}\right)^{2 n} P_{2 n}(0) \quad \text { for } r<a .
$$

\section{Conclusion}

We discussed the spatial distribution of electrical field generated by a uniformly charged disc in the spherical coordinates frame. By solving mathematical physics problem of the uniformly charged ring, we get the general solution of the equation. Taking into accoun the boundary condition,we get the spatial distribution potential of the ring. Using the superposition principle of potential, we can obtain the electric potential of a uniformly charged disc, and figure the three-dimensional potential distribution image. Using this method, we can have a clear physical picture about the physical problem, understand the problem more easily and accomplish without complex mathematical calculation. Furthermore, by using the equation $E=-\nabla u$, we can easily obtain the spatial distribution electrical field of the charged disc from Eq. (20).

\section{References}

Cao Yujuan, Dong Shenxing. (2005). The electric field on a plane generated by uniformly charged circle. Physics and Engineering, 15, p12 14.

K. F. Riley, M. P. Hobson and S. J. Bence. (2002). Mathematical methods for physics and engineering (second edition). Cambridge: Cambridge University Press. P143, 676.

LI Qing-yu, SHAN Yu-qiong. (2002). The electric potentical on evenly electrified thin disc. Journal of Yunnan Normal University, 22, p39 40.

N. S. Koshlyakov, M. M. Smirnov, \& E. B. Gliner. (1964). Differential Equations of Mathematical Physics. New York: Interscience (Wiley). p205.

R. Courant, D. Hilbert. (1966). Methods of Mathematical Physics. New York: Intersicence Publishers, Inc. p503.

Zhou Hai-ying, Chen Hao. (2004). Space distribution of electrical field generated by a uniformly charged ring. Collge Physics, 23, p32 34.

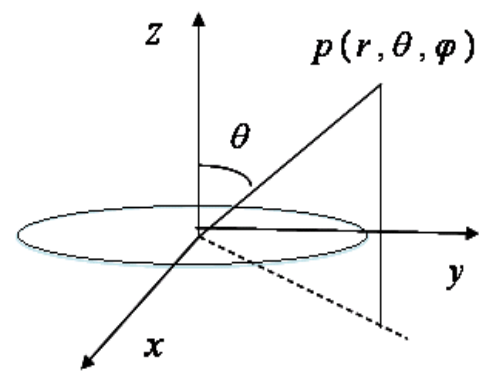

Figure 1. Position of the Thin Charged Ring in the Spherical Coordinates System 


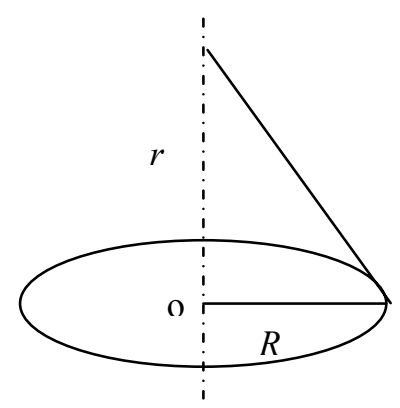

Figure 2. Electric Potential on the Axis of the Ring

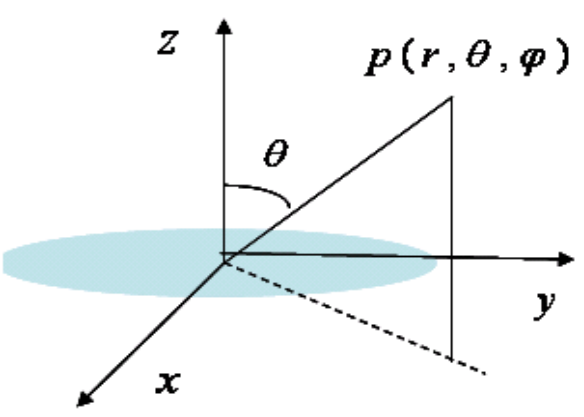

Figure 3. A Uniformly Charged Disc with Coordinates for Finding the Potential

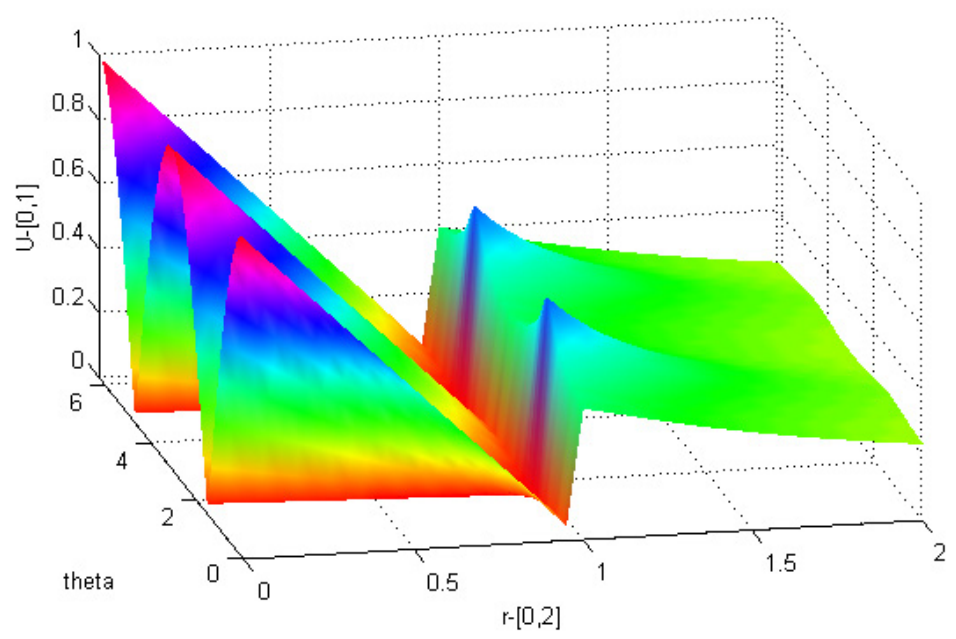

Figure 4. Three-Dimensional Potential Distribution Image of the Charged Disc 